\title{
POSITIVITY OF STABLE DENSITIES
}

\author{
S. C. PORT AND R. A. VITALE
}

(Communicated by Bert E. Fristedt)

\begin{abstract}
We settle two conjectures of Taylor about the positivity of the densities $p(t, x)$ of a drift-free, nondegenerate, stable process on $d$-dimensional Euclidean space $R^{d}$ starting at the origin. If $0<\alpha<1$ and $p(1,0)=0$, we show that $\{x: p(t, x)>0$ for some $t>0\}$ is an open convex cone with vertex 0 and that $p(t, x)>0$ for all $t>0$ for each $x$ in this cone. If $\alpha=1$ we show that $p(t, x)>0$ for all $t>0$ and all $x \in R^{d}$.
\end{abstract}

A drift-free stable process $X(t)$ on $d$-dimensional Euclidean space starting at the origin is a process having stationary independent increments with log characteristic function of the form $-\lambda t|z|^{\alpha} \int W_{\alpha}(z, \theta) \mu(d \theta)$, where $\lambda>0$ and $\mu$ is an arbitrary finite nonzero measure on the unit sphere. The parameter $\alpha \in(0,2]$, and

$$
\begin{aligned}
W_{\alpha}(z, \theta) & =\left[1-i \operatorname{sgn}(z \cdot \theta) \tan \left(\frac{\pi \alpha}{2}\right)\right]\left|\frac{z}{|z|} \cdot \theta\right|^{\alpha}, \quad \alpha \neq 1, \\
& =\left|\frac{z}{|z|} \cdot \theta\right|+\frac{2 i}{\pi}\left(\frac{z}{|z|} \cdot \theta\right) \ln |z \cdot \theta|, \quad \alpha=1 .
\end{aligned}
$$

We will call the measure $\mu$ the sphere measure of the process and let $X(t)$ be the associated $d$-dimensional process. Let $S(\mu)$ be the support of $\mu$. If $S(\mu)$ lies on a great circle of the sphere (i.e. if $S(\mu)$ is on a hyperplane through the origin) the distribution of $X(t)$ is degenerate. Otherwise, it has a bounded continuous density $p(t, x)$. Let $d_{0}$ be the dimension of the smallest subspace containing $S(\mu)$. Then the process may be viewed as one on $R^{d_{0}}$ where it is nondegenerate and thus has a bounded continuous density. Our purpose in this paper is to investigate the positivity properties of $p(t, x)$ and to settle two conjectures of Taylor [2] about these densities.

In [2] Taylor classified nondegenerate stable processes into two types. A process is type $A$ iff $p(1,0)>0$; otherwise the process is type $B$. In [1] Port showed that every nondegenerate process of Type A with $\alpha \neq 1$ has the property that $p(t, x)>0$ for all $t>0$ and all $x \in R^{d}$. Taylor [2] showed that for $\alpha \neq 1$ a nondegenerate stable process is Type B iff $0<\alpha<1$ and $S(\mu)$ is contained in some closed hemisphere. He also showed that the interior $K$ of the closed convex cone with vertex 0 generated by $S(\mu)$ is exactly $\{x: p(t, x)>0$ for some $t>0\}$. He conjectured that in fact $p(t, x)>0$ for all $t>0, x \in K$. Our first theorem asserts that this conjecture is correct.

Received by the editors June 12, 1986 and, in revised form, January 30, 1987. 1980 Mathematics Subject Classification (1985 Revision). Primary 60E10, 60J30.

Key words and phrases. Stable distributions, stable densities, stable processes.

Partially supported by NSF Grant DMS 8603944. 
THEOREM 1. Let $0<\alpha<1$ and assume $S(\mu)$ is contained in a hemisphere and does not lie on a great circle. Then the stable process on $R^{d}$ having sphere measure $\mu$ has densities $p(t, x)$ on $R^{d}$ such that $p(t, x)>0$ for all $t>0$ for each $x$ in the interior $K$ of the convex cone with vertex 0 generated by $S(\mu)$. For $x \notin K$, $p(t, x)=0$ for all $t>0$.

The processes with $\alpha=1$ are called Cauchy processes. It is known that all such processes in $R^{1}$ have densities that are strictly positive for all $x$ and $t>0$. In [2] Taylor conjectured that this was also the case in $R^{d}$. Our second result is that this conjecture is true.

THEOREM 2. Let $X(t)$ be a nondegenerate Cauchy process on $R^{d}$. Then the densities $p(t, x)>0$ for all $t>0$ and all $x \in R^{d}$.

Years ago one of the authors told the late J. Folkman of Taylor's first conjecture. Folkman informed that author that he thought he could prove the conjecture by a geometric argument using induction on the dimension, but supplied no further details. Since our proof does not proceed by induction and uses the processes in an essential way it seems that it must be different from what Folkman had in mind. ship

PROOF OF THEOREM 1 . The densities $p(t, x)$ satisfy the basic scaling relation-

$$
p(t, x)=p\left(r t, r^{1 / \alpha} x\right) r^{d / \alpha}
$$

for all $r, t>0$ and $x \in R^{d}$. They also satisfy the semigroup property

$$
p(t+s, z)=\int p(t, z-x) p(s, x) d x
$$

for all $z, t>0$. Let $S(t)=\{x: p(t, x)>0\}$ and let $S=S(1)$. Then (1) shows that $S(t)=t^{1 / \alpha} S$. Now (2) and the continuity of the densities show that for all $t, r>0$

$$
S(t+r)=S(t)+S(r) \text {. }
$$

Combined, we find that for all $t, r>0$

$$
(t+r)^{1 / \alpha} S=t^{1 / \alpha} S+r^{1 / \alpha} S .
$$

It follows from (4) and $S(t)=t^{1 / \alpha} S$ that $K=\bigcup_{t>0} S(t)$ is an open convex cone with vertex 0 . Taylor [2] has shown that this cone is exactly the interior of the closed convex cone with vertex 0 generated by $S(\mu)$. The assertion of Theorem 1 is equivalent to $S=K$. This will follow at once if we can show that $S$ is a cone. Note that under the assumptions of Theorem $1, p(1,0)=0$. By $(1), p(t, 0)=p(1,0) t^{-d / \alpha}$. Hence $0 \notin K$.

Now $\bar{K}$, the closure of $K$, is a closed cone contained in some closed half-space. We will call a closed cone $C^{*}$-pointed iff $C \backslash\{0\}$ is contained in an open half-space. Our first result is that if Theorem 1 holds for all processes such that $\bar{K}$ is *-pointed, then it holds for all processes satisfying the assumptions of Theorem 1.

LEMMA 1. Assume that the assertions of Theorem 1 are valid for processes such that $\bar{K}$ is ${ }^{*}$-pointed. Then Theorem 1 holds in general.

PrOOF. As before $S(\mu)$ is the support of $\mu$. By hypothesis, it is not contained on a great circle (i.e. $S(\mu)$ does not lie on a hyperplane through the origin). 
Consequently, $S(\mu)$ has at least $d$ points. Pick $x_{1}, \ldots, x_{d-1} \in S(\mu)$. These determine a unique great circle. There must be another point $x_{d} \in S(\mu)$ not on this great circle. Hence $x_{1}, \ldots, x_{d}$ lie on no great circle and thus the plane through $x_{1}, \ldots, x_{d}$ cannot contain the origin. Hence $x_{1}, \ldots, x_{d}$ are in some open hemisphere as is the closure of a small (spherical) neighborhood $D_{1}$ of $\left\{x_{1}, \ldots, x_{d}\right\}$. Let $\mu_{1}$ be the restriction of $\mu$ to $D_{1}$ and let $\bar{K}_{1}$ be the closed convex cone generated by $S\left(\mu_{1}\right)$. Then $\bar{K}_{1}$ is ${ }^{*}$-pointed and the process having sphere measure $\mu_{1}$ is nondegenerate. Let $D_{2}, \ldots, D_{r}$ be other sets such that $D_{i} \cap S(\mu) \neq \varnothing, D_{1}, \ldots, D_{r}$ partition $S(\mu)$, and each $\bar{D}_{i}$ lies in some open hemisphere (generally depending on $i$ ). Let $\mu_{i}$ be the restriction of $\mu$ to $D_{i}$ and let $\bar{K}_{i}$ be the convex cone generated by $S\left(\mu_{i}\right)$. Then each $\bar{K}_{i}$ is ${ }^{*}$-pointed. It can happen however that the process $X_{i}(t)$ having sphere measure $\mu_{i}$ is degenerate. Assume that processes $X_{2}, \ldots, X_{r_{0}}$ are nondegenerate and that processes $X_{r_{0}+1}, \ldots, X_{r}$ are degenerate. Since the $\mu_{i}$ are concentrated on disjoint sets and $\mu=\sum \mu_{i}$ the processes $X_{1}, \ldots, X_{r}$ are independent, and $X(t) \stackrel{D}{=} X_{1}(t)+\cdots+X_{r}(t)$. By assumption Theorem 1 holds for the processes $X_{1}, \ldots, X_{r_{0}}$. Let $\bar{J}$ be the convex cone generated by $S\left(\mu_{1}+\cdots+\mu_{r_{0}}\right)$, $J$ the interior of $\bar{J}$. Let $Y(t)=X_{1}(t)+\cdots+X_{r_{0}}(t)$. Then $Y(t)$ has a density $q(t, x)$ and each $X_{i}(t)$ has a density $p_{i}(t, x)$. If $y \in J$ then $q(t, y)>0$ for some $t$. Hence $\exists x_{1}, \ldots, x_{r_{0}}$ such that $p_{i}\left(t, x_{i}\right)>0$ and $x_{1}+\cdots+x_{r_{0}}=y$. Since Theorem 1 holds for each $X_{i}, 1 \leq i \leq r_{0}, p_{i}\left(s, x_{i}\right)>0$ for all $s>0$ and hence $q(s, y)>0$ for all $s>0$. Thus Theorem 1 is valid for the process $Y(t)$. Consider the process $X_{i}$ for $i=r_{0}+1, \ldots, r$. Let its degenerate distribution on $R^{d}$ be $F_{i}(t, d x)$ and let $d_{i}$ be the dimension of the smallest subspace containing $K_{i}$. Then $\bar{K}_{i}$ is a *-pointed cone in $R^{d_{i}}$ and the process on $R^{d_{i}}$ having sphere measure $\mu_{i}$ is nondegenerate. Consequently, by our assumption it has a density that is positive for all $t$ for all points in $K_{i}$, the interior of $\bar{K}_{i}$. But then, $\bar{K}_{i}$ is the support of $F_{i}(t, d x)$ for all $t>0$. Let $Z(t)=X_{r_{0}+1}(t)+\cdots+X_{r}(t)$. Since all $X_{i}(t)$ have common support for all $t$, the distributions $F(t, d x)$ of $Z(t)$ also have common support for all $t$. Now $X(t) \stackrel{D}{=} Y(t)+Z(t)$ and $Y, Z$ are independent. Let $z \in K$. Then $p(t, z)>0$ for some $t$. Since

$$
p(t, z)=\int q(t, z-x) F(t, d x),
$$

it must be that there is an $x$ in the support of $F(t, d x)$ such that $q(t, z-x)>0$. But then $q(s, z-x)>0$ for all $s$, and $x$ is in the support of $F(s, d x)$ for all $s$. Hence $p(s, z)>0$ for all $s>0$. This shows that Theorem 1 holds in general.

The proof of the *-pointed case is based on three lemmas. As a preliminary to the first, we consider the special case of (4) with $r+t=1$. This shows that for $0 \leq r \leq 1$

$$
r^{1 / \alpha} S+(1-r)^{1 / \alpha} S=S .
$$

Let $x \in S$. Then (5) shows $\left[r^{1 / \alpha}+(1-r)^{1 / \alpha}\right] x \in S$. Since $\alpha<1,\left[r^{1 / \alpha}+(1-r)^{1 / \alpha}\right]$ has a minimum in $(0,1)$. Hence there is a $\lambda_{0}, 0 \leq \lambda_{0}<1$, such that $\lambda x \in S$ for all $\lambda, \lambda_{0} \leq \lambda \leq 1$. By iteration, $\lambda x \in S$ for all $0<\lambda \leq 1$. Let $\bar{S}$ be the closure of $S$. It easily follows that

$$
r^{1 / \alpha} \bar{S}+(1-r)^{1 / \alpha} \bar{S} \subseteq \bar{S}
$$


and proceeding as above we find that if $x \in \bar{S}$, then the closed segment connecting $x$ to 0 is also in $\bar{S}$.

LEMMA 2. Let $T$ be a subset of the half-line $[0, \infty)$ satisfying the relationship

$$
r^{1 / \alpha} T+t^{1 / \alpha} T=(r+t)^{1 / \alpha} T, \quad r, t>0 .
$$

Then either $T$ is empty, $\{0\}$ or $T \supset(0, \infty)$.

Proof. Assume $\exists x \neq 0$ in $T$. Then $\lambda x \in T$ for all $0<\lambda \leq 1$. Suppose $T$ is bounded above. Let $a$ be its least upper bound. Take $y \in T$ such that $2^{1 / \alpha} y>2 a$. Taking $r=t=1$ we see that $T+T=2^{1 / \alpha} T$. Hence $\exists x, z \in T$ such that $x+z=2^{1 / \alpha} y$. But $x \leq a, z \leq a \Rightarrow x+z \leq 2 a$, a contradiction. Hence $T$ is not bounded and thus $T \supseteq(0, \infty)$.

The projection of $S$ onto any ray is a one-dimensional set having the required property of Lemma 2. This fact implies that $S$ and indeed any nonvoid intersection of $S$ with a half-space must contain points of arbitrarily large norm.

LEMMA 3. Let $\mu$ satisfy the requirements of Theorem 1 and assume additionally that $\bar{K}$ is $a^{*}$-pointed cone. Then $\bar{S}$ is a closed convex cone.

ProOF. For $n=1,2, \ldots$, let

$$
A_{n}=\{x /|x|: x \in \bar{S},|x| \geq n\} .
$$

Our discussion above implies that $A_{n}$ is nonempty. We observe that if $x \in \bar{S}$ then $\lambda x \in \bar{S}$ for all $\lambda \in[0,1]$. It follows that we may rewrite $A_{n}$ as

$$
A_{n}=\{x /|x|: x \in \bar{S},|x|=n\} \text {. }
$$

It easily follows from this representation that $A_{n}$ is compact. Since the sequence $A_{1}, A_{2}, \ldots$ is nested decreasing, there is a common point $e \in A=\bigcap_{n} A_{n}$. But then $n e \in \bar{S}$ for all $n=1,2, \ldots$, and thus the ray $\{\lambda e\} \subseteq \bar{S}$. Consequently, the set $S^{*}$ of all rays in $\bar{S}$ is nonempty. We will next show that it is a closed convex cone. The argument above shows that the closed cone generated by $A$ contains $S^{*}$ and obviously $S^{*}$ contains the closed cone generated by $A$. Hence $S^{*}$ is the closed cone generated by $A$. As to convexity it follows from (6) that if $x, y \in \bar{S}$ then so does the arc $r^{1 / \alpha} x+(1-r)^{1 / \alpha} y, 0 \leq r \leq 1$. Thus, if two rays lie in $S^{*}$, then all arcs, and hence all line segments connecting them, lie in $S^{*}$.

It remains to show that $S^{*}=\bar{S}$, or what is the same that $\bar{S} \subseteq S^{*}$. Suppose that there is an $x \in \bar{S}$ that is not in $S^{*}$. In deriving a contradiction we show there is a separating hyperplane $H^{*}$ (through the origin) which places $x$ in one open half-space and $S^{*} \backslash\{0\}$ is in the other open half-space. Since $S^{*} \subseteq \bar{S} \subseteq \bar{K}$ and $\bar{K}$ is ${ }^{*}$-pointed, $S^{*}$ is ${ }^{*}$-pointed. Its spherical image $A$ thus lies in an open hemisphere, and by assumption it is at a positive distance from $x /|x|$. Then a standard separation result yields the desired hyperplane $H^{*}$, which supports $S^{*}$ and which places $x$ in an open half-space of $H^{*}$. It follows that $S^{*} \backslash\{0\}$ lies in the other open half-space. Let $H^{+}$be the half-space containing $x$. Observe that $H^{+} \cap S \neq \varnothing$ and consequently

$$
B_{n}=\left\{y /|y|: y \in H^{+} \cap \bar{S},|y| \geq n\right\}
$$

is nonempty for all $n=1,2, \ldots$. The $B_{n}$ form a nested decreasing sequence of nonempty compact sets and arguing as with the $A_{n}$ we can conclude that $\overline{H^{+}}$ contains a ray in $\bar{S}$. But $\overline{H^{+}} \cap S^{*}=\{0\}$ which is a contradiction. Hence $S^{*}=\bar{S}$. 
LEMMA 4. The interior of $\bar{S}$ is $S$.

ProOF. Let $S_{0}$ be the interior of $\bar{S}$. To show that $S=S_{0}$, or equivalently, $S_{0} \subseteq S$ it is enough to show that any $z \in S_{0}$ can be written as

$$
z=r^{1 / \alpha} x+(1-r)^{(1 / \alpha)} y
$$

for some $r \in(0,1)$ and $x, y \in S$. With such a $z$ given, fix $x_{0} \in S$ and define

$$
y(x)=(1-r)^{-1 / \alpha} z-(r /(1-r))^{1 / \alpha} x .
$$

Since $\lim _{r \rightarrow 0}(1-r)^{-1 / \alpha} z-(r / 1-r)^{1 / \alpha} x=z$ and $z \in S_{0}$, it must be the case that $y\left(x_{0}\right) \in S_{0}$ for some $r>0$, which we now fix in (8). Observe that $y(\cdot)$ maps an open ball centered at $x_{0}$ onto a ball of center $y\left(x_{0}\right) \in S_{0} \subseteq \bar{S}$. Thus the image ball must contain a point $y(\hat{x})$ of $S$. Since $S$ is open, we can choose a ball of center $x_{0}$ all of whose points are in $S$. Then $\hat{x}$ and $y(\hat{x})$ satisfy $(7)$.

PROOF OF THEOREM 1 . Since $\bar{S}$ is a convex cone, its interior $S_{0}$ is a convex cone. Lemma 4 shows $S=S_{0}$. Hence $S$ is a convex cone, and this establishes Theorem 1 for $\bar{K}$ a $^{*}$-pointed cone. Lemma 1 now shows that the statement of Theorem 1 is true in general.

Proof OF ThEOREM 2. Let $a=\int \theta \mu(d \theta)$. The densities of the Cauchy processes satisfy the scaling relationship.

$$
p(t, x)=t^{-d} p(1, x / t-((2 / \pi) \ln t) a) .
$$

Let $S(t)=\{x: p(t, x)>0\}$ and let $S=S(1)$. Then it follows from (9) that

$$
S(t)=t S+a(2 / \pi) t \ln t .
$$

The semigroup property shows that for $t_{1}, \ldots, t_{m}>0$

$$
S\left(t_{1}+\cdots+t_{m}\right)=S\left(t_{1}\right)+\cdots+S\left(t_{m}\right) .
$$

From (10) and (11) we find

$$
S=t_{1} S+\cdots+t_{m} S+\left(\frac{2}{\pi} \sum_{i=1}^{m} t_{i} \ln t_{i}\right) a
$$

for all $t_{i}>0, \sum_{i=1}^{m} t_{i}=1$. Let $b=(-(2 / \pi) \ln m) a$ and

$$
g\left(t_{1}, \ldots, t_{m}\right)=-\sum_{i=1}^{m} t_{i} \ln t_{i} / \ln m .
$$

Then (12) is the same as

$$
S=\sum_{i=1}^{m} t_{i} S+g\left(t_{1}, \ldots, t_{m}\right) b
$$

or, equivalently,

$$
\sum_{i=1}^{m} t_{i} S=S-g\left(t_{1}, \ldots, t_{m}\right) b .
$$

Let $m=d+1$. Carathéodory's theorem then shows that the union over all $t_{1}, \ldots, t_{m}, t_{i} \geq 0, \sum t_{i}=1$ of the sets on the LHS of (14) is just the convex hull of 
$S(\operatorname{conv}(S))$. Since $g$ is continuous with maximum 1 and minimum 0 the union over all such $t_{i}$ of the sets on the RHS of (14) is just $S-L$, where $L=\{\lambda b: 0 \leq \lambda \leq 1\}$. Hence

$$
\operatorname{conv}(S)=S-L
$$

Since $L$ is compact $\overline{S-L}=\bar{S}-L$. Thus

$$
\overline{\operatorname{conv}(S)}=\bar{S}-L \text {. }
$$

Now the projection of $S$ onto any line is the entire line. Indeed, for any unit vector $e$ the process $e \cdot X_{1}$ is a one-dimensional Cauchy variable, and hence has strictly positive density. This density is produced by integrating $p(1, x)$ over the hyperplane through 0 orthogonal to $e$. Thus for any point $x$ in the space spanned by $e$ there must be points in $S$ whose projection onto this space is $x$. Hence the projection of $\overline{\operatorname{conv}(S)}$ onto any line is the entire line. This implies that $\overline{\operatorname{conv}(S)}=R^{d}$. [For suppose $\exists x \notin \overline{\operatorname{conv}(S)}$. There is then a support hyperplane such that $\overline{\operatorname{conv}(S)}$ is in one half-space and $x$ is in the interior of the other half-space. But then the projection of $\overline{\operatorname{conv}(S)}$ onto the line in the direction of the normal to support hyperplane through $x$ cannot be the entire line.] From (16) we conclude that $\bar{S}-L=R^{d}$. Using (13) for $m=2$ we find that if $x \in S$, then so does $x+g\left(t_{1}, t_{2}\right) b$ for all $t_{1}, t_{2} \geq 0, t_{1}+t_{2}=1$. As $g$ has maximum 1 at $t_{1}=t_{2}=\frac{1}{2}$ and minimum 0 at $t_{1}=0$ or 1 , we can conclude that $S+L \subseteq S$. Thus $\bar{S}+L \subseteq \bar{S}$. Let $z \in R^{d}$. Since $\bar{S}-L=R^{d}$, we can find $\lambda \in[0, \overline{1}]$ so that $z+\lambda b \in \bar{S}$. Hence $z+b=(z+\lambda b)+(1-\lambda) b \in \bar{S}$. Thus $R^{d}=R^{d}+b \subseteq \bar{S}$. Hence $R^{d}=\bar{S}$.

We finally show that $S=R^{d}$. Let $x \in S, z \in R^{d}$, and let

$$
y(x)=\frac{z}{1-r}-\frac{r}{1-r} x-\frac{g(r)}{1-r} b,
$$

where $g(r)=-((r \ln r)+(1-r) \ln (1-r)) / \ln 2$. Then

$$
r x+(1-r) y+g(r) b=z \text {. }
$$

Let $x_{0} \in S$. The function $y$ in (17) maps a ball of center $x_{0}$ onto a ball of center $y\left(x_{0}\right)$. Since $\bar{S}=R^{d}$ there must be a point of $S$ in the image ball. Hence by selecting a sufficiently small ball about $x_{0}$ (recall $S$ is open) we obtain points $x, y(x) \in S$ satisfying (18). Then (13) for $n=2$ shows $z \in S$. Hence $S=R^{d}$.

Query. Our proof of Theorem 1 heavily uses the basic property (4). Using that property and a strictly geometric argument we showed that if $\bar{S}$ is known to be contained in a ${ }^{*}$-pointed cone, then $S$ itself is a convex cone. The general case depends on Lemma 1 which uses further properties of the process. It would be of interest to have a purely geometric proof. That is, if $S$ is an open subset of $R^{d}$ and satisfies (4) for all $r, t>0$, is $S$ a convex cone with vertex 0 ? The argument used in [1] to establish Theorem 1 shows that if $0 \in S$ and $S$ satisfies (4), then $S=R^{d}$. Thus the case when $0 \notin S$ and $S$ is not contained in a $*$-pointed cone awaits a purely geometric argument.

\section{REFERENCES}

1. S. C. Port, A remark on hitting places for transient stable processes, Ann. Math. Statist. 39 (1968), 365-371.

2. S. J. Taylor, Sample path properties of transient stable processes, J. Math. Mech. 16 (1967), 1229-1246.

Department of Mathematics, University of California, los Angeles, CaliFORNIA 90024 